\title{
Multiple Tuberous Xanthomas: A Diagnostic Dilemma on Cytology
}

\author{
Richa Bhartiya ${ }^{1 *}$, Pallavi Agrawal ${ }^{1}$, Rajnish Kumar ${ }^{1}$ and Rajendra Prasad Dwivedi ${ }^{2}$ \\ 'Dept. of Pathology, Patna Medical College \& Hospital (PMCH), Ashok Rajpath, Patna Bihar India \\ ${ }^{2}$ Department of Pathology, Govt. Medical College, Betiah (Paschim Champaran), Bihar, India
}

\begin{abstract}
Tuberous xanthomas are large nodular lesions that develop in patients with underlying hyperlipoproteinemias. A patient was referred to us for fine needle aspiration cytology (FNAC) from multiple lesions over extensor aspect of upper and lower limbs, which yielded scanty aspirate despite several attempts. This case is being reported to emphasize how such lesions can present as diagnostic challenge on cytology, the role of which has not clearly been described in the previous case reports.
\end{abstract}

\section{Keywords: Xanthoma, FNAC, Hyperlipoproteinemias, Cytology.}

\section{Introduction}

Xanthoma is a localized collection of tissue histiocytes containing lipid. It is not a true tumour but, rather, a reactive histiocyte proliferation that occurs in response to alterations in serum lipids. ${ }^{[1]}$ Xanthomas mostly develop in primary and secondary hyperlipoproteinemias and occasionally in normolipemic states. Usually, xanthomas occur in skin and subcutis, but occasionally involve deep soft tissue such as tendons or synovium. ${ }^{[2]}$ Herein, we present a case of multiple tuberous xanthomas diagnosed on Fine Needle Aspiration Cytology (FNAC). This case is being reported to emphasize the challenge faced while diagnosing such lesions on FNAC. Also most of the previous reports have not mentioned the role of cytology in diagnosing these lesions.

\section{Case Report}

A 21-year old female presented to the medicine outpatient department with a history of multiple nodular lesions over bilateral elbow, knee and foot for the past five years. The lesions first appeared over the extensor aspect of bilateral elbow joints followed by similar nodules over bilateral ankle joints, dorsal aspect of the foot and lastly over bilateral knee joints. Otherwise the patient was healthy with no family history of similar lesions in parents or siblings. There was no history of diabetes, hypothyroidism or any other systemic disease in the patient. On physical examination, multiple yellowish, firm, non-tender cutaneous lesions were identified over the extensor aspect of bilateral elbow, ankle and knees, largest measuring 2.5 $\mathrm{cm}$ in size over the right elbow (Fig 1, 2 \& 3). FNAC was performed from multiple sites. One of the attempts yielded whitish aspirate while rest were blood mixed. Smears were sparsely cellular and showed predominantly foamy histiocytes in clusters and scattered singly, few benign mesenchymal cells and occasional inflammatory cells in a hemorrhagic background(Fig 4). She had a normal haemogram. Her biochemical investigations showed normal blood glucose levels, liver function tests, kidney function tests and electrolytes levels, however the lipid profile was deranged and showed elevated Cholesterol (391 mg\%) and LDL (331 mg\%) levels.

Correlating with the biochemical findings, a possibility of Xanthoma was considered on cytology and an excision (Fig 5) and histopathological correlation was advised. Histopathological examination from one of the lesions revealed infiltration of dermis by foamy histiocytes along with giant cells, fibrosis and cholesterol clefts thus confirming the diagnosis (Fig 6, 7 \& 8).

\section{Discussion}

Xanthomas are infiltrates of the skin that are yellow to brown red in colour and result from infiltration of the dermis by lipid containing cells. Xanthomas may be a part of general metabolic disease. ${ }^{[3]}$ Tuberous xanthomas present as discrete, occasionally multilobated painless nodules or large plaque like lesions of the subcutis. Xanthomas may be divided into several categories: Tendinous xanthoma, xanthoma tuberosum, eruptive xanthoma, xanthoma planum, and palmar xanthoma. ${ }^{[4]}$ The most commonly xanthomas among patients with familial hypercholesterolemia are tendinous xanthomas, which are subcutaneous tumours located within the tendons used for extension. ${ }^{[5,6]}$ They usually present over pressure areas such as extensor aspect of elbows, knees and buttocks. ${ }^{[3,7]}$ A previous study has shown that the most frequent site for 


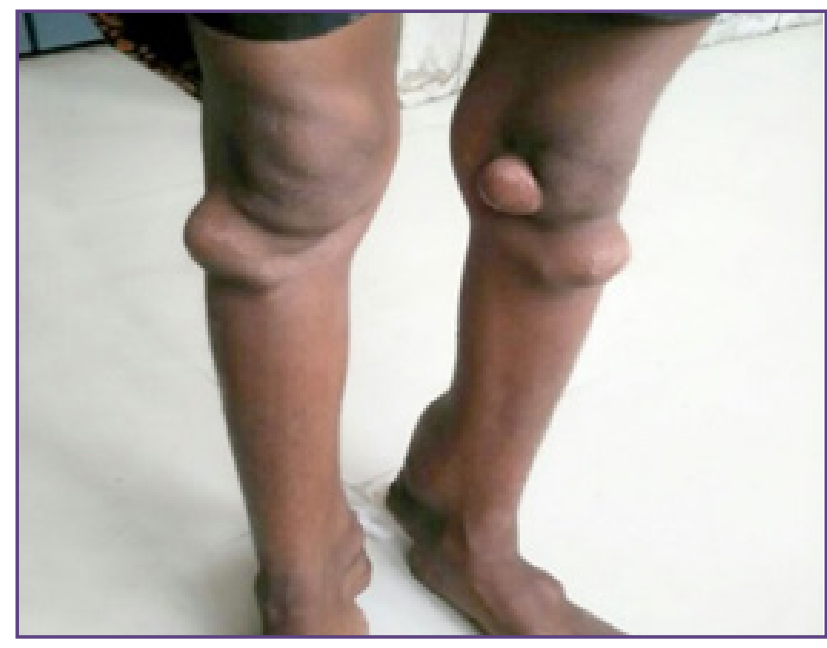

Fig. 1; Bilateral knees.

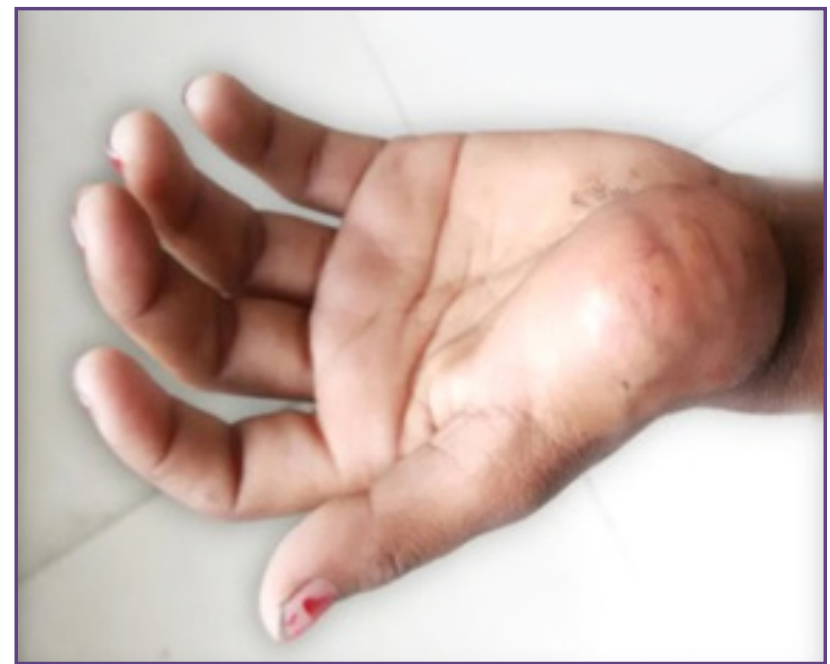

Fig. 3: Left palm.

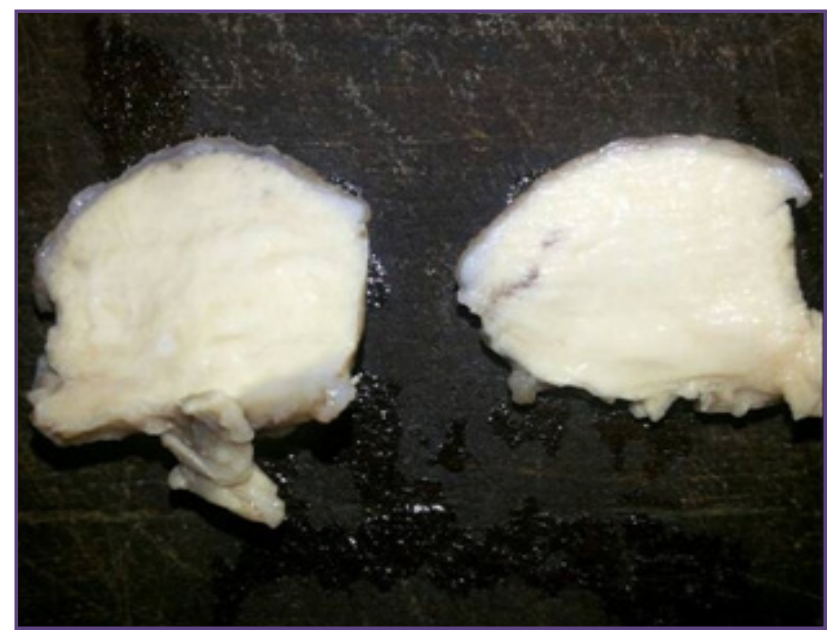

Fig. 5: Gross image: excised left elbow xanthoma: skin covered nodule with shiny yellowish cut surface.

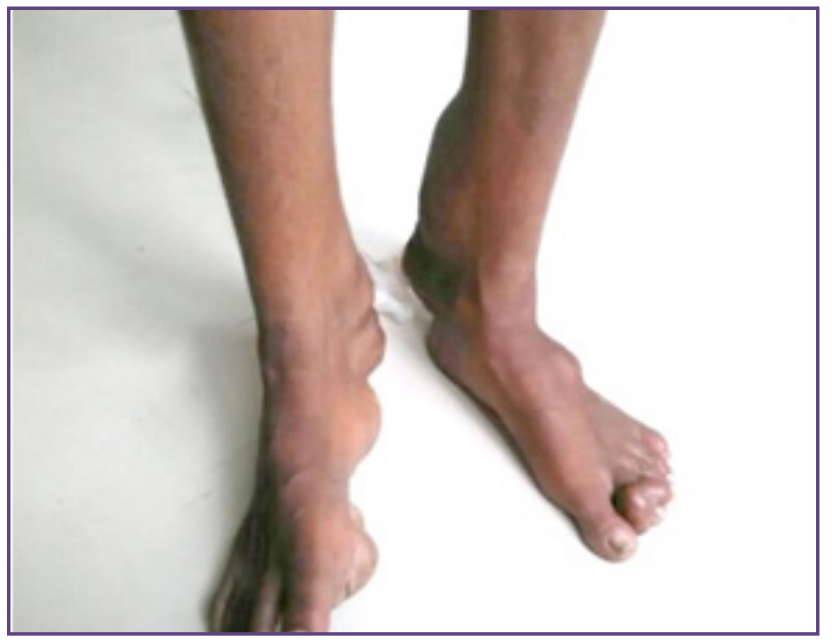

Fig. 2: Foot.

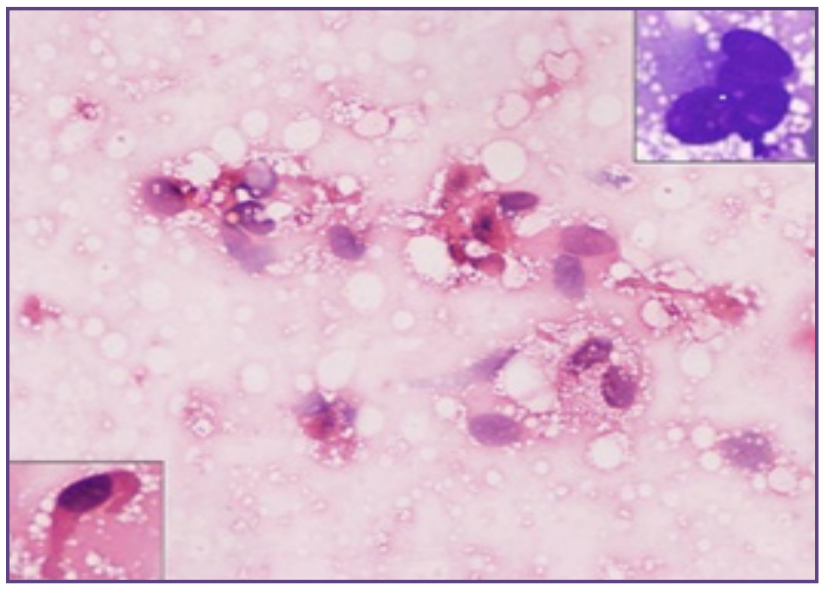

Fig. 4: FNAC smear, Haematoxylin and Eosin (H\&E, 40X): smears show clusters of foamy histiocytes in a proteinaceous background; Upper right inset: giant cell; Lower left inset: mesenchymal cell.

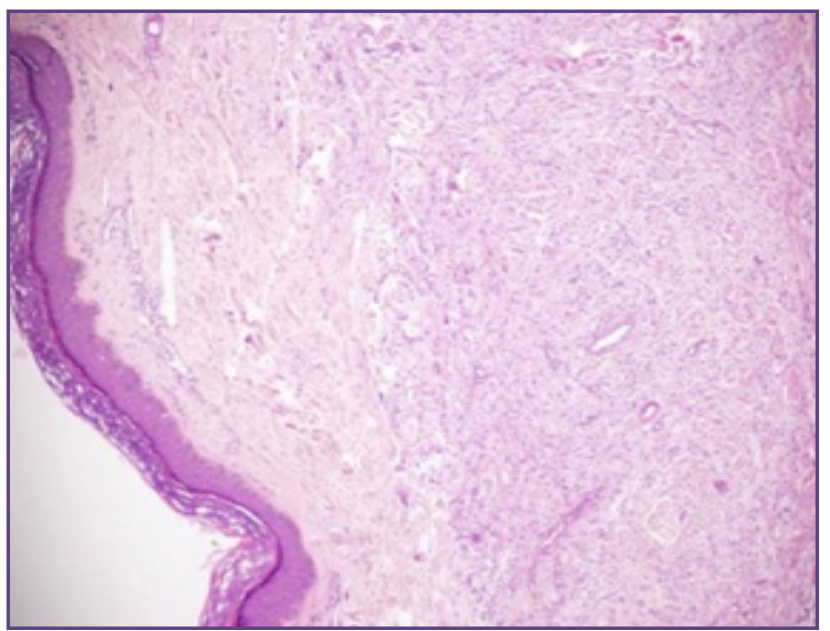

Fig. 6: H\&E, 4X: Shows infiltration of dermis by large collections of foam cells. 


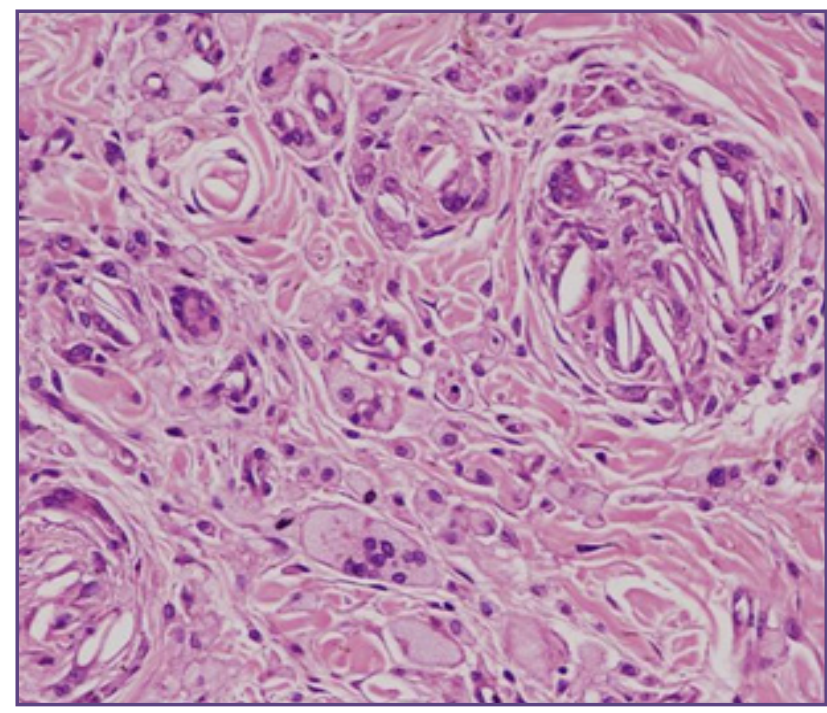

Fig. 7: H\&E, 20X: Higher magnification shows foamy macrophages, cholesterol clefts, giant cells, collagenous tissue and few inflammatory cells in the dermis.

xanthomas is the Achilles' tendon. ${ }^{[8]}$ Xanthomas consist of cholesterol, cholesterol esters, triglycerides, phospholipids and numerous lipid-laden foamy macrophages. ${ }^{[9]} \mathrm{case}$, the patient had lesions at similar sites. Hyperlipidemias may basically be classified as either familial (also called primary caused by specific genetic abnormalities, or acquired (also called secondary) when resulting from another underlying disorder that leads to alterations in plasma lipid and lipoprotein metabolism. Also, hyperlipidemia may be idiopathic, that is, without known cause. Hyperlipidemias are also classified according to which types of lipids are elevated, that is hypercholesterolemia, hypertriglyceridemia or both in combined hyperlipidemia. Familial hyperlipidemias are classified according to the Fredrickson classification ${ }^{[10]}$ which is based on the pattern of lipoproteins on electrophoresis or ultracentrifugation. Tuberous xanthomas are usually associated with type IIA or type III hyperlipoproteinemias as well as secondary hyperlipoproteinemias. In our case too, the patient had Type II A Hypercholesterolemia.

Cutaneous xanthomas are designated according to their appearance and clinical presentation. Eruptive xanthomas are small, yellow papules developing in individuals with hyperlipoproteinemia types I, III, and V. Tuberous xanthomas are large plaque-like lesions of the subcutis, usually located on the buttocks, elbows, knees, and fingers and are seen with type IIa or III hyperlipoproteinemia. Plane xanthomas occur in skinfolds, such as the palmar creases, and are characteristic of type III hyperlipoproteinemia. Occasionally they occur in normolipemic persons. Xanthelasmas are xanthomas of the eyelid and usually

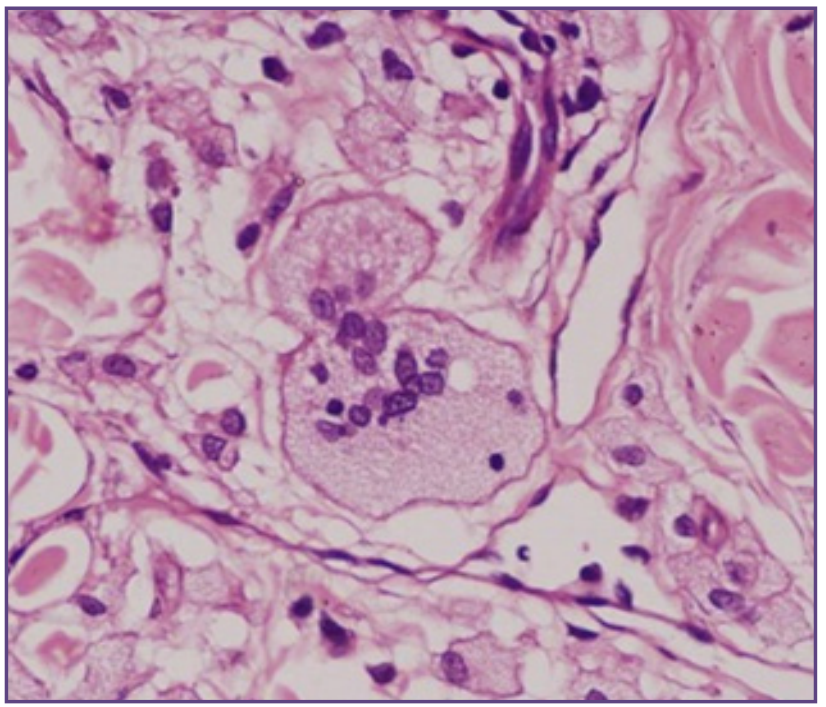

Fig. 8: H\&E, 40X: Section shows multinucleated giant cell and foamy macrophages in the dermis.

are observed in normolipemic persons, although they also occur in those with type IIa or III hyperlipoproteinemia.

Previously, xanthomas were considered as a neoplastic lesion, but their association with hyperlipidemic states confirms that these are benign reactive lesions. ${ }^{[1]}$ The pathogenesis suggested in such lesions are that the lipid in these lesions is derived from blood. ${ }^{[1]}$ The serum lipoproteins leave the vascular compartment, traverse small vessels, and enter the macrophages of soft tissue. Once ingested by macrophages the lipoprotein is degraded to lipid, and the lipid is released to the extracellular space. The fibrosis characteristic of mature or longstanding xanthomas is believed to be related to the fibrogenic properties of extracellular cholesterol. This series of events can be confirmed ultrastructurally by the sequential finding of lipoprotein between endothelium and basement membrane and finally in the pericytes. Although xanthomas can potentially occur at any soft tissue site, the localization stimulus seems directly related to the vascular permeability, as agents that increase permeability (e.g., histamine) can accelerate xanthoma formation at a given site. Likewise, minor trauma or injury that results in histamine release also accelerates xanthoma formation. This observation provides an explanation for the common occurrence of such lesions in the tendons of the hands and feet which would also be the probable cause in our case. Foamy macrophages are commonly found in cutaneous lesions. Fibrohistiocytic tumors, such as dermatofibroma and atypical fibroxanthoma, can show prominent "lipidization", but other characteristics of these tumors allow differentiation from primary xanthomas. Diagnostic 
difficulty generally occurs between dermatofibroma and tuberous xanthoma, but the latter shows foam cells and dense collagenization, without the prominent cellular component of dermatofibroma.In atypical fibroxanthomas, the spindle cell component shows pleomorphism and is usually seen in head and neck region of elderly people.In our case, despite several attempts from different lesions, FNAC yielded scant aspirate. Cytosmears were sparsely cellular with predominantly foamy histiocytes and few benign mesenchymal cells. A differential diagnosis of benign fibrohistocytic lesion and xanthoma was considered just on cytomorphology. Benign fibrohistiocytic lesion was considered in the differential as few mesenchymal cells were seen along with the histiocytes. Biochemical investigations revealed an abnormal lipid profile and helped in clinching a diagnosis of xanthoma. Histopathological examination of one of the excised lesions showed infiltration of dermis by foamy macrophages along with cholesterol clefts, fibrosis and few giant cells thus confirming a diagnosis of xanthoma. Zhao et al also reported such a case in a 23-year old male having multiple large xanthomas with familial hypercholesterimia in which histopathological examination of excised lesion along with bio-chemical findings confirmed the diagnosis, however, they have not attempted the fine needle aspiration. ${ }^{[12]}$

\section{Conclusion}

Tuberous xanthomas may present as a diagnostic challenge on FNAC due to several reasons such as low yield, lack of specific cytological features as well as features common with other lesions. However, in the right clinical setting and with the help of biochemical investigations, such a lesion should be kept in mind while making a diagnosis on cytology thus emphasizing its role in diagnosing such lesions. Prompt diagnosis and treatment may help to prevent side-effects such as early coronary artery disease and pancreatitis.

\section{Reference}

1. Marcoval J, Moreno A, Bordas X, et al. Diffuse plane xanthoma: clinicopathologic study of 8 cases. J. Am Acad Dermatol. 1998;39:439.

2. FaheyJJ, StarkHH, Donovan WF, etal.Xanthoma of the Achilles tendon: seven cases with familial hyperbetalipoproteinemia. J Bone Joint Surg Am. 1973;55:1197.

3. Singh AJ, Sikarwar S, O P Jatav, and Saify K. Normolipemic tuberous xanthomas.Indian J Dermatol. 2009 (AprJun);54(2):176-179.

4. Zak A, Zeman M, Slaby A and Vecka M: Xanthomas: Clinical and pathophysiological relations. Biomed Pap Med Fac Univ Palacky Olomouc Czech Repub 158: 181-188, 2014.

5. Sethuraman G, Sugandhan S, Sharma G, Chandramohan K, Chandra NC, Dash SS, Komal A and Sharma VK: Familial homozygous hypercholesterolemia: Report of two patients and review of the literature. Pediatr Dermatol 24: 230-234, 2007.

6. AlvesCandBraidZ:Homozygousfamilialhypercholesterolemia: Case report of a rare cause of dyslipidemia. Pediatr Endocrinol Diabetes Metab 17: 162-165, 2011.

7. Babu R, Venkataram A, Santhosh S, Shivaswamy S. Giant tuberous xanthomas in a case of Type II A Hypercholesterolemia. J Cutan Aesthet Surg. 2012 (JulSep);5(3):204-206.

8. Dagistan E, Canan A, Kizildag B and Barut AY: Multiple tendon xanthomas in patient with heterozygous familial hypercholesterolaemia: Sonographic and MRI findings. BMJ Case Rep 2013: 2013.

9. Szalat R, Arnulf B, Karlin L, Rybojad M, Asli B, Malphettes M, Galicier L, Vignon-Pennamen MD, Harel S, Cordoliani F, et al: Pathogenesis and treatment of xanthomatosis associated with monoclonal gammopathy. Blood 118: 3777-3784, 2011.

10. DonaldSFredrickson, RobertSLees.Asystemforphenotyping hyperlipoproteinemia. Circulation 1965;31(3):321-7.

11. Iton KW, Thomas C, Dunkerley DJ: The pathogenesis of xanthomata. J Pathol 1973;109:271.

12. Zhao $C$ et al. Multiple large xanthomas: A Case Report. Oncology Letters 2016, 12 (6): 4327-4332

*Corresponding author:

Dr Richa Bhartiya, Dept. of Pathology, Patna Medical College \& Hospital (PMCH), Ashok Rajpath,Patna Bihar-800004 India

Phone: +91 07738237073

Email: richabhartiya1972@gmail.com

Financial or other Competing Interests: None.

Date of Submission : 13.03.2017

Date of Acceptance : 14.08.2017

Date of Publication : 12.12.2017 